\title{
The APS upgrade offering new opportunities for materials science U. Ruett ${ }^{1}$ \\ ${ }^{1}$ Argonne National Laboratory uruett@anl.gov
}

The Advanced Photon Source has played a major role in the materials science research for 25 years. Now the source will be upgraded starting in April 2023. In 2024, users can expect an ultra-bright source operated at $6 \mathrm{GeV}$ with high fraction of coherence even at high-energy x-ray. There will be 9 new feature beamlines built to take full advantage of the new source parameter, and many beamlines will become enhanced including insertion devices, optics, and instrumentation.

11-ID-D operated by the Structural Science group at the APS is one of the enhanced beamlines, which will enable a combination of total scattering with small angle scattering and focusing into the submicrometer range. Here, we can close the gap between the resolution in reciprocal and real space to provide a complete picture of the structure of materials. Multimodal setups and photon energies between $26 \mathrm{keV}$ and $120 \mathrm{keV}$ with highest flux will enable complex in situ and operando experiments. The main emphasis will be on the understanding and discovery of new materials covering in situ synthesis and manufacturing to studies during functionality.

In this talk, upcoming opportunities for research in materials science at the upgraded APS will be discussed. 